\title{
Development of fault section identification technique for low voltage DC distribution systems by using capacitive discharge current
}

\author{
Chul-Ho NOH ${ }^{1}$, Chul-Hwan KIM ${ }^{1}$, Gi-Hyeon GWON ${ }^{1}$, \\ Yun-Sik $\mathbf{O H}^{1}$
}

\begin{abstract}
The increasing importance of energy efficiency has led to several studies related to the construction of a reliable low voltage DC (LVDC) distribution system. Specifically, studies on a protection scheme that considers the fault characteristics of an LVDC distribution system are essential to improve system reliability. When compared to a conventional distribution system, the most distinct feature of an LVDC distribution system is the existence of a capacitive discharge current from converters under a fault condition that results in the prompt operation of protection devices. Therefore, this study involves proposing a precise and rapid technique to identify the fault section in an LVDC distribution system. The technique involves two stages, namely: an analysis stage to analyze the capacitive discharge current and a decision stage to identify the fault section based on the fault type. A detailed discussion of each step is presented and its feasibility is verified based on the results of simulations with an ElectroMagnetic Transients Program and MATLAB ${ }^{\circledR}$.
\end{abstract}

CrossCheck date: 24 October 2017

Received: 10 December 2016/ Accepted: 24 October 2017 / Published online: 5 January 2018

(C) The Author(s) 2018. This article is an open access publication

Chul-Hwan KIM

chkim@skku.edu

Chul-Ho NOH

chcoo87@skku.edu

Gi-Hyeon GWON

elysium03@skku.edu

Yun-Sik OH

fivebal2@skku.edu

1 College of Information and Communication Engineering, Sungkyunkwan University, Suwon, Republic of Korea
Keywords Capacitive discharge current, Fault section identification, Fault type identification, Low voltage DC distribution system

\section{Introduction}

Low voltage DC (LVDC) distribution systems are expected a solution to problems encountered by typical power systems in terms of energy efficiency, power loss, and interconnections with distributed generation (DG) systems. However, there is a paucity of studies related to the construction of a reliable LVDC distribution system due to differences when compared to conventional systems and especially in relation to its structural characteristics $[1,2]$. According to the Computer Business Equipment Manufacturers Association (CBEMA) curve, sensitive components are damaged by voltage that corresponds to $70 \%$ of its rated value even if it only lasts for $100 \mathrm{~ms}[3,4]$. This means that an LVDC distribution system requires a rapid and precise protection scheme because it includes many sensitive components such as converters and digital loads. Specifically, the rapid identification of the fault section or location is directly related to the reliability of an LVDC distribution system [5, 6].

Three factors should be considered while developing a technique to identify a fault in an LVDC distribution system. First, the fault characteristics of an LVDC distribution system are considered. When a fault occurs in an LVDC distribution system, a capacitive discharge current begins to flow from the DC-link capacitor inside the AC/DC converter [7, 8]. This current displays a distinct feature of the fault, and thus it is necessary to perform an analysis of the capacitive discharge current during the transient state immediately after the fault. Thus, Prony's method can be 
utilized for fault analysis as shown in previous studies $[9,10]$.

However, the anti-parallel diodes can be damaged after the DC-link capacitor is fully discharged due to the discharge of line inductor and voltage source [11-13]. This fault characteristic results in extremely fast fault detection and interruption compulsory for an LVDC distribution system. Therefore, it is impossible to analyze current by using Prony's method due to the lack of data with respect to capacitive discharge current. In order to overcome this challenge, an additional back-up capacitor [3] or probe power unit [14-17] are installed.

Another consideration is that the technique to identify the fault section should consider structural differences from the transmission system such as the existence of multiple branches and short line lengths. An extremely significant problem related to the structure corresponds to the possibility of a multi-estimation problem as a result of the branches. Hence, this limits the use of representative techniques such as an impedance-based method [18, 19]. In the case of travelling wave-based methods, the multi-estimation problem is solved by using high frequency components [20-22]. However, the accuracy of these methods is still decreased because the travelling wave is disturbed by branch [23].

Therefore, the present study proposes a novel technique to identify the fault section of an LVDC distribution system. This technique is based on the characteristics of the capacitive discharge currents from DC/DC converters. The current also depicts the information on fault conditions even within a period of milliseconds similar to that from AC/DC converter. Additionally, the structural features of a DC/DC converter provide a powerful advantage in which it is possible to gather sufficient information from the capacitive discharge current without additional devices such as a probe power unit [24]. Furthermore, the method eliminates any potential multi-estimation problem. This is because it is necessary to install DC/DC converters in front of each load in the LVDC distribution system to convert the voltage level for customers. Specifically, this technique targets a ring-type LVDC distribution system that is utilized as a microgrid interconnecting a DG system and energy storage system (ESS) [25, 26].

The rest of this paper is organized as follows. Section 2 presents the characteristics of the capacitive discharge current according to the fault conditions in an LVDC distribution system. This is followed by discussing the concept of the developed technique and the details of each step of the algorithm in Sect. 3. Section 4 describes the results of simulations conducted under various conditions using an electromagnetic transients program (EMTP) and MATLAB ${ }^{\circledR}$ to verify and demonstrate the feasibility of the proposed technique. Finally, the conclusions are presented in Sect. 5.

\section{Characteristics of capacitive discharge current based on fault conditions}

This section analyzes the characteristics of the capacitive discharge current from a DC/DC converter in an LVDC distribution system based on both the fault location and type. First, the concept of a ring-type LVDC distribution system is introduced. The transferred AC voltage is rectified to a DC voltage by an $\mathrm{AC} / \mathrm{DC}$ converter, and then the converted DC voltage is distributed to customers through distribution lines. Especially, the distribution lines form in ring type and are distributed to customers in the middle. The distributed DC voltage is lowered to the voltage level required by customers through a DC/DC converter. Especially, distribution line forms in ring type.

This is followed by discussing the generation of the capacitive discharge current from the DC/DC converter. The power semiconductor switch in DC/DC converter is switched off immediately after a fault occurs on the distribution line. Naturally, a series RLC circuit is formed as shown in Fig. 1, and these results in the discharge of the input capacitor [7-9]. During this period, the capacitive discharge current flows in the series RLC circuit.

\subsection{Characteristics according to fault location}

According to an extant study [24], the capacitive discharge current that generally includes an underdamped sinusoidal waveform $i_{\text {fault }}(t)$ is expressed as:

$i_{\text {fault }}(t)=-\exp \left(-\left(R_{e q} / L_{e q}\right) t\right) \sqrt{\left(I_{0}\right)^{2}+B^{2}} \sin \left(\omega_{d} t+\theta\right)$

where $R_{e q}$ and $L_{e q}$ denote the equivalent resistance and inductance, respectively; $\theta$ presents the angle determined by parameters of fault loop; $I_{0}$ denotes the initial current; $B=\left(2 V_{C}-R_{e q} I_{o}\right) / 2 L_{e q} \omega_{d}, \quad$ and $\quad \omega_{d}=$ Root $\quad\left[\left(L_{e q-}\right.\right.$ $\left.C)^{-1}-\left(R_{e q} / 2 L_{e q}\right)^{2}\right]$.

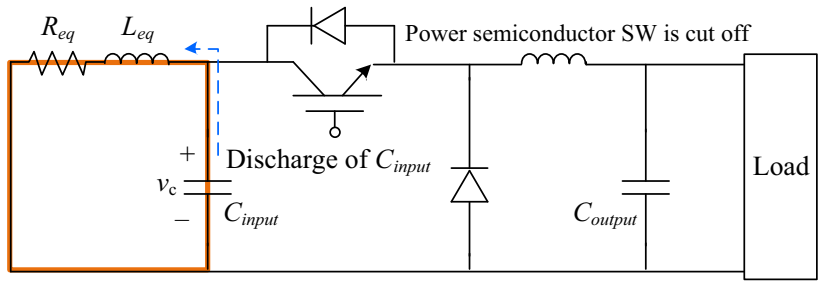

Fig. 1 Internal structure of DC/DC converter and fault loop resulting from the opening of power semiconductor switches 
The capacitive discharge current depends on the equivalent impedance that is determined by the fault location. Thus, the information on a fault is obtained by analyzing the capacitive discharge current. The current consists of three terms as shown in (1), namely exponential, constant, and sine terms. Although there is an increase in the fault distance that corresponds to a length between fault location and DC/DC converter, the exponential term remains constant because the $R_{e q}$ and $L_{e q}$ increase proportionally at the same rate. This means that the exponential term that represents the damping factor is constant irrespective of the fault location.

Conversely, the sine term and constant term are affected due to the damped radian frequency $\omega_{d}$ and $B$ of the constant term, respectively. In the case of the sine term, the denominator of $\omega_{d}$ is proportional to the fault distance. Additionally, $R_{e q}$ and $L_{e q}$ change at the same rate based on fault distance. However, the numerator is inversely proportional to fault distance. Therefore, the $\omega_{d}$ is inversely proportional to the fault distance. In the case of the constant term, both denominator and numerator of $B$ decrease with increases in fault distance. However, $B$ is also inversely proportional to fault distance because the change in the rate of the numerator of $B$ exceeds that of the denominator.

As previously mentioned, capacitive discharge current highly depends on fault distance in the aspects of magnitude and frequency. Thus, the proposed technique significantly considers these types of characteristics of the capacitive discharge current to identify the exact fault section.

\subsection{Characteristics according to fault type}

In a bipolar LVDC distribution system, various types of faults can occur on a distribution line including a pole-toground (PTG) fault, a pole-to-neutral (PTN) fault, and a pole-to-pole (PTP) fault [27]. A PTG fault is the same as a ground fault in an $\mathrm{AC}$ system, and the other two types of faults correspond to a type of short circuit fault. In this section, the characteristic of the capacitive discharge current is analyzed according to fault type in two aspects, namely magnitude and polarity.

The magnitude of the capacitive discharge current is inversely proportional to the fault distance as discussed in Sect. 2.1. The capacitive discharge current resulting from a PTP fault displays the fore-mentioned characteristic. In contrast, in the case of the other two fault types, the connection method for the DC/DC converter is another factor that affects the magnitude of the capacitive discharge current because of the change in the fault loop parameters $R_{e q}, L_{e q}$, and $C_{e q}$. Table 1 shows the values according to
Table 1 Fault loop parameters based on fault type

\begin{tabular}{llll}
\hline Fault type & \multicolumn{2}{l}{ Fault loop parameter } & \\
\cline { 2 - 4 } & $R_{e q}$ & $L_{e q}$ & $C_{e q}$ \\
\hline (+) PTG fault & $(2-\alpha) R_{\text {line }}$ & $(2-\alpha) L_{\text {line }}$ & $C$ \\
(-) PTG fault & $(2+\alpha) R_{\text {line }}$ & $(2+\alpha) L_{\text {line }}$ & $C / 3$ \\
(+) PTN fault & $2(1-\alpha) R_{\text {line }}$ & $2(1-\alpha) L_{\text {line }}$ & $C$ \\
(-) PTN fault & $2 R_{\text {line }}$ & $2 L_{\text {line }}$ & $C / 3$ \\
\hline
\end{tabular}

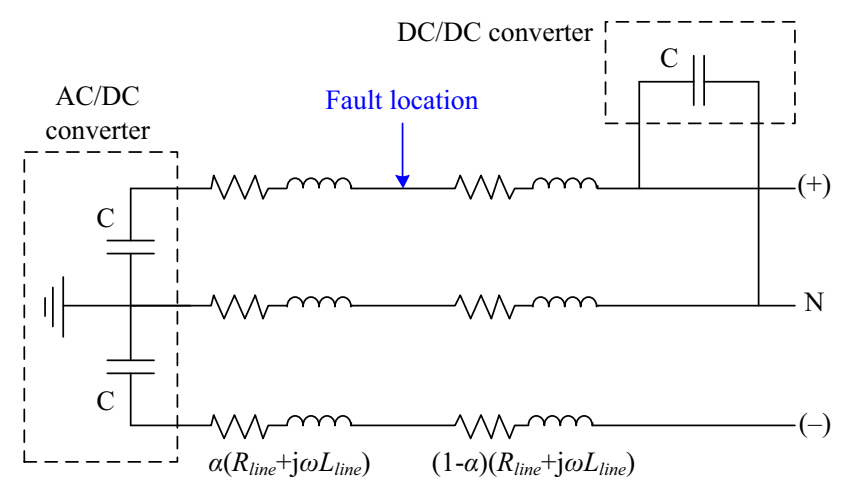

Fig. 2 A simplified circuit diagram in the case of the DC/DC converter connecting with a $(+)$ pole-neutral line

fault type based on Fig. 2 with the exception of a PTP fault. It is assumed that fault resistance corresponds to zero.

As shown in Table $1, R_{e q}$ and $L_{e q}$ exhibit a relative increase when a fault occurs on opposite pole from that the $\mathrm{DC} / \mathrm{DC}$ converter is connected to. The increase in $R_{\mathrm{eq}}$ or $L_{e q}$ decreases the magnitude of capacitive discharge current as discussed in Sect. 2.1. Additionally, a decrease of $C_{e q}$ results in a decrease in the magnitude. In conclusion, the magnitude of the capacitive discharge current must be low despite the short fault distance when a PTG or PTN fault occurs on the opposite pole. This characteristic is used to distinguish a PTG fault from a PTP fault in the proposed algorithm.

The polarity of the capacitive discharge current at the beginning of the transient state is also a distinct characteristic that can distinguish the fault type and specifically a PTN fault. When a fault loop is formed, capacitive discharge current must flow from a higher voltage conductor to a lower voltage conductor. When a PTG or PTP fault occurs, then the capacitive discharge current flows out along the path (1) and flows in along with the path (2) as shown in Fig. 3a. However, when a PTN fault occurs, the capacitive discharge current from a DC/DC converter connected to a healthy pole flows in the opposite direction. That is because a fault loop is formed through the fault between neutral line and negative pole as shown in Fig. $3 b$. In this case, the capacitive discharge current flows out 


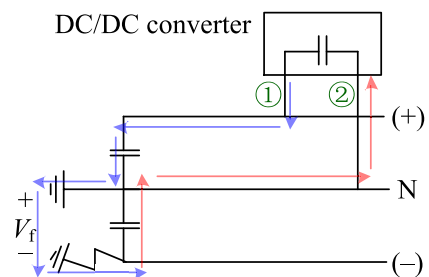

(a) PTG fault

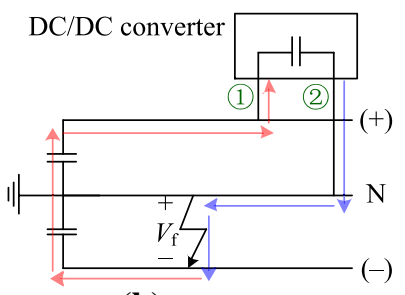

(b) PTN fault
Fig. 3 Flow of capacitive discharge current by a fault on the opposite conductor from the pole connected with a DC/DC converter

along the path (2) because of the fault voltage $V_{\mathrm{f}}$ in Fig. $3 \mathrm{~b}$. Thus, this characteristic is related to polarity and is used to identify a PTN fault.

As shown in this section, the three fault types can be distinguished based on two clear characteristics. This process is essential because the proposed algorithm to identify the precise fault section in an LVDC distribution system uses different methods based on the fault type.

\section{Proposed technique fault section identification}

\subsection{Review of conventional methods}

Conventional methods to identify a fault section in a ring-type distribution system are based on the fault current as opposed to voltage along the feeder [15, 28]. This is because it is possible to specify a fault section more accurately by measuring the flow direction of a fault current. When the fault section in a radial distribution system is identified, then it is acceptable to approximately estimate the fault section based on the characteristic wherein the voltage along the feeder decreases. Nevertheless, in the case of a ring-type distribution system, system reliability is directly affected by identifying the precise fault section. Thus, the method used with respect to the direction of fault current is reasonable in the case of a ring-type distribution system. In a conventional AC system, the phase difference is used to distinguish between a reference voltage and the fault current [29, 30]. In contrast, in a DC system, the polarity of the fault current is easily used as the distinguishing factor.

The method with the current direction continues to be subject to a significant issue related to the amount of sensors in a distribution system. Additionally, high quality sensors are required to avoid the case in which the estimation error by the capacitive discharge current results from an underdamped sinusoidal form for dozens of milliseconds after the occurrence of a fault. Furthermore, the analyzed data in all the sections of the LVDC distribution system should be exchanged with each other through a communication system. Given these drawbacks, this study proposes a novel technique for fault section identification in a ring-type LVDC distribution system.

The proposed technique for a ring-type LVDC distribution system is based on the characteristics of capacitive discharge current from a DC/DC converter according to fault conditions. The technique does not require additional sensors to measure and analyze capacitive discharge currents because the fore-mentioned processes are performed in each DC/DC converter. Hence, the proposed technique can identify a precise fault section without the installation of additional sensors in the target system when compared to conventional methods that use current direction and voltage along the feeder.

\subsection{Concept of proposed technique}

The study involves proposing the technique to identify the fault section in a ring-type LVDC distribution system, and Fig. 4 shows the concept of the proposed technique.

When a fault occurs in distribution line, it is possible to measure capacitive discharge current at the DC/DC converter. The capacitive discharge current is analyzed at each converter in two aspects. The first aspect corresponds to the output process in which outputs including two types of data related to the magnitude and polarity are obtained by analyzing the capacitive discharge current. The other aspect corresponds to the sorting process to determine the necessity of the analyzed data based on convexity of the capacitive discharge current. With respect to the standard for convexity, DC/DC converters transfer the analyzed data to a converter station as shown in Fig. 4. The transferred data is analyzed with respect to the converter station, and the control center identifies the fault section in the LVDC distribution system. This is followed by sending the operation signal to the corresponding protective devices to isolate the fault section as shown in Fig. 4. Given that only the minimum fault section is isolated through the proposed technique, all customers do not experience an outage as long as a ring-type system is used.

\subsection{Identification algorithm of the proposed technique}

This section shows the detailed algorithm for the proposed technique to identify the fault section in a ring-type LVDC distribution system. As shown in Fig. 5, the algorithm consists of two stages, namely the analysis stage and decision stage. The analysis stage corresponds to the analysis of capacitive discharge current at the $\mathrm{DC} /$ DC converter and is composed of the following four steps: sampling, decomposition, and analysis (reducing and identifying parts). The decision stage is conducted in 


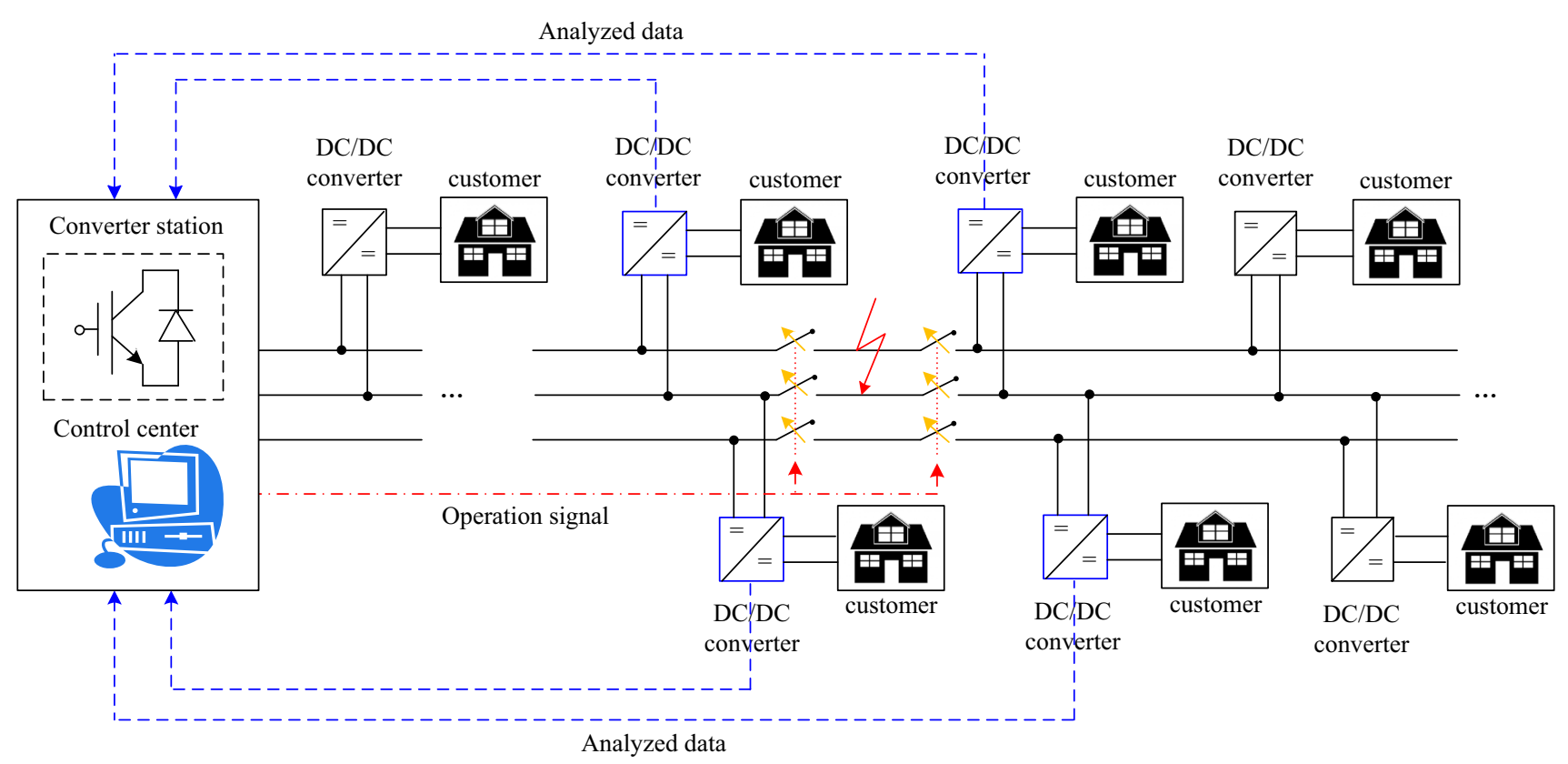

Fig. 4 Concept of the proposed technique

the control center to identify the fault section. In this stage, the identification of the fault section follows the classification of fault type. The detailed procedure is as follows.

\subsubsection{Analysis stage: sampling}

In the proposed technique, current at all the converters is sampled in real time without additional sensors. In this aspect, it is necessary to set a sampling frequency to enable the analysis of the capacitive discharge current. Generally, the specific minimum value of sampling frequency is set through a system study. However, in the proposed technique, the sampling frequency is set equally with the switching frequency of the DC/DC converter. That is because the setting is sufficient to cover the requirement for analyzing the capacitive discharge current and it does not require additional design considerations for the DC/DC converter to analyze the capacitive discharge current. In this step, a moving window technique is applied to calculate $\mathrm{d} i / \mathrm{d} t$ and $\mathrm{d}^{2} i / \mathrm{d} t^{2}$ of the capacitive discharge current as well as use Prony's method.

\subsubsection{Analysis stage: decomposition}

In this step, the sampled signal is divided into two parts, namely the reducing part and identifying part. This step is initiated when $\mathrm{d} i / \mathrm{d} t$ exceeds the set threshold value $\delta$ as shown in (2). The time satisfying condition (2) is designated as $t_{\text {int }}$ as: $|\mathrm{d} i / \mathrm{d} t|>\delta$

The sign change in $\mathrm{d} i / \mathrm{d} t$ at the pole is used to divide the capacitive discharge current as shown in (3). The times when the condition (3) is satisfied first and second are designated as $t_{r e d}$ and $t_{i d}$, respectively. The character $r$ in (3) indicates the order to be sampled. This is expressed as follows:

$\mathrm{d} i /\left.\mathrm{d} t\right|_{r-1} \cdot \mathrm{d} i /\left.\mathrm{d} t\right|_{r}<0$

Based on the time mentioned above, the reducing part (from $t_{\text {int }}$ to $t_{\text {red }}$ ) and the identifying part (from $t_{\text {red }}$ to $t_{i d}$ ) are extracted from the sampled signal as shown in Fig. 6.

\subsubsection{Analysis stage: analysis on reducing part}

The reducing part is analyzed by using two factors, namely $\mathrm{d} i / \mathrm{d} t$ and $\mathrm{d}^{2} i / \mathrm{d} t^{2}$. The analysis using the first factor $(\mathrm{d} i / \mathrm{d} t)$ is conducted simultaneously with the step of decomposition, and a polarity $\left(m_{l}\right)$ of the capacitive discharge current is compared with an allocated polarity $\left(n_{l}\right)$ at any DC/DC converter $(\# l)$. The $n_{l}$ indicates the polarity of the conductor connected with a specific DC/DC converter. The output data $\left(S_{l}\right)$ results from a comparison between $m_{l}$ and $n_{l}$. Table 2 summarizes the manner in which the three values are determined. As mentioned in Sect. 2.2, a PTN fault must reverse the direction of capacitive discharge current such that $S_{l}$ is used to distinguish a PTN fault.

The second factor $\left(\mathrm{d}^{2} i / \mathrm{d} t^{2}\right)$ is used to evaluate the convexity of the reducing part. In a typical distribution system, travelling wave can be disturbed by branches [31]. Hence, the reducing part becomes convex down as a fault is 


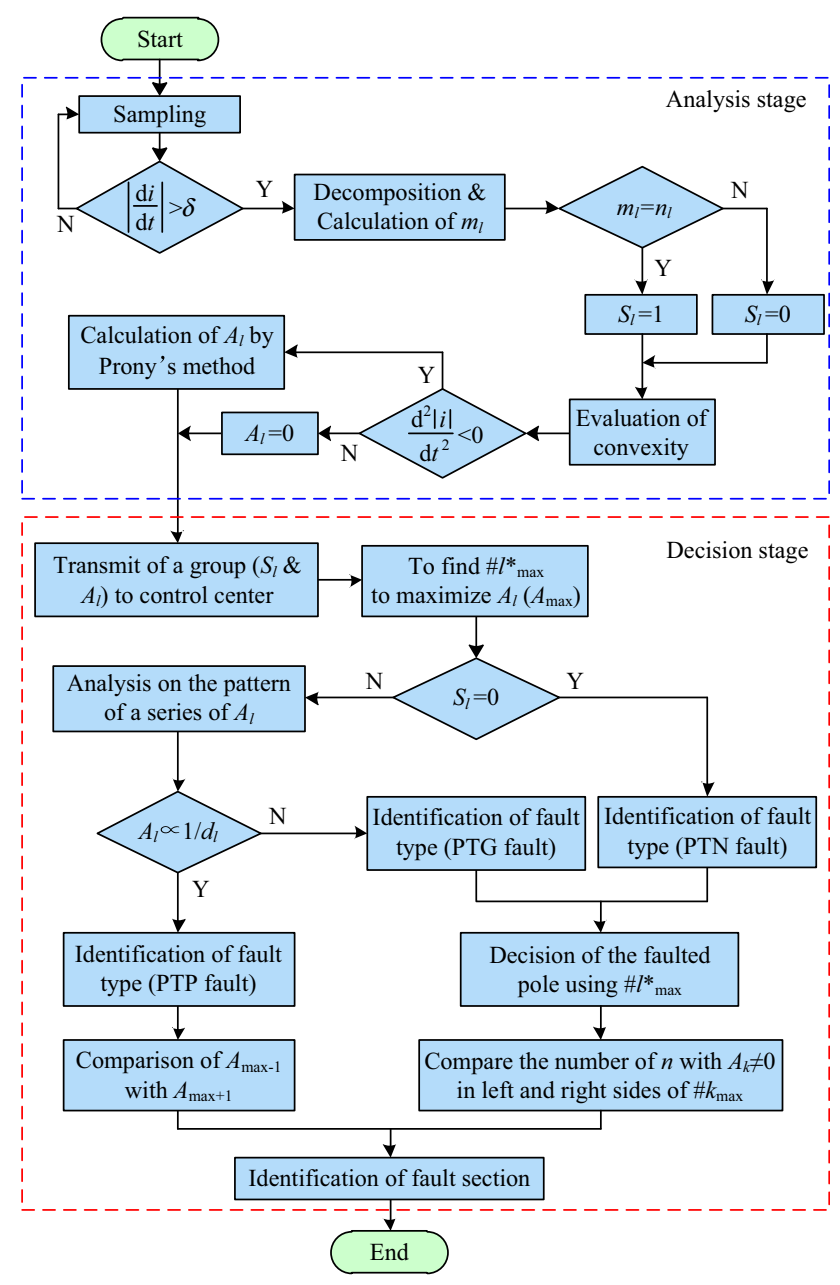

Fig. 5 Algorithm of the proposed fault section identification technique

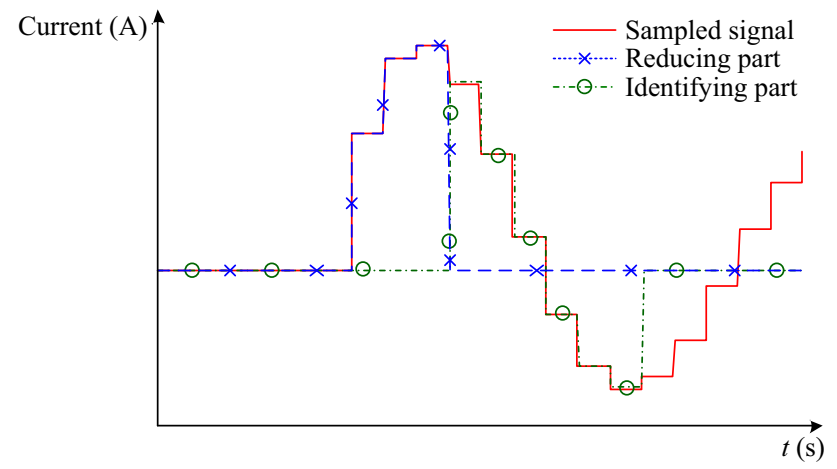

Fig. 6 Decomposition of sampled signal

located at a distance. On the other hand, the current is convex up resulting from the adjacent fault. In the proposed technique, the output data acquired from DC/DC converters adjacent to the fault location is sufficient to identify the exact fault section. In this regard, convexity of
Table 2 Fault loop parameters based on fault type

\begin{tabular}{llr}
\hline Parameter & Condition & Value \\
\hline$m_{l}$ & $\mathrm{~d} i / \mathrm{d} t>0$ & 1 \\
& $\mathrm{~d} i / \mathrm{d} t<0$ & -1 \\
$n_{l}$ & $\# l$ with $(+)$ pole & 1 \\
& $\# l$ with $(-)$ pole & -1 \\
$S_{l}$ & $m_{l}=n_{l}$ & 1 \\
& $m_{l} \neq n_{l}$ & 0 \\
\hline
\end{tabular}

the capacitive discharge current corresponds to the criterion used to determine the necessity of DC/DC converters. If (4) is satisfied, with respect to the convex up, further analysis on the identifying part proceeds and the corresponding DC/DC converter is represented by "*” (for e.g. $\left.\# l^{*}\right)$. If not, the DC/DC converters simply transfers the meaningless value. This is expressed as:

$\mathrm{d}^{2}|i| / \mathrm{d} t^{2}<0$

\subsubsection{Analysis stage: analysis on identifying part}

Prony's method is a signal processing technique that is used to analyze the transient states in a power system by fitting a linear combination of exponential terms to a signal as shown in (5) [32]. Given that (1) corresponds to the same equation as (5) with $q=1$, the simplified Prony's method is extremely useful in analyzing capacitive discharge current in an LVDC distribution system.

$x(t)=\sum_{k=1}^{q} A_{k} \exp \left(\Delta \delta_{k} t\right) \cos \left(2 \pi f_{k} t+\theta_{k}\right)$

where $q$ denotes the number of elementary functions, which corresponds to the sum of the purely damped exponentials and the sinusoids whether damped or not [33]. Additionally, there are four real factors, namely the frequency $f_{k}$ in hertz, damping factor $\delta_{k}$, magnitude $A_{k}$, and phase $\theta_{k}$ in radians. Extracting $N$ samples with a $\Delta t$ sampling period from the measured signal, (5) can be rewritten as (6) using Euler's rule.

$x_{n}=\sum_{k=1}^{p} \beta_{k} z_{k}^{n} \quad n=0,1, \ldots, N-1$

where $p$ denotes the signal order, which corresponds to the sum of the purely damped exponentials and complex terms plus their conjugates [33]. In (6), two complex factors are found, namely complex magnitude $\beta_{k}$ and complex frequency $z_{k}$. The relation between the real factors in (5) and 
complex factors in (6) is expressed as (7) and (8). The $k$ in (7) and (8) is limited as $q$ because the other values of $p$ and corresponding conjugates output the same results. This is expressed as:

$$
\begin{aligned}
\beta_{k} & =\frac{1}{2} A_{k} e^{\mathrm{j} \theta_{k}} \\
z_{k} & =e^{-\left(\delta_{k}+\mathrm{j} 2 \pi f_{k}\right) \Delta t}
\end{aligned}
$$

A detailed procedure of Prony's method is explained in a previous study [33]. In this step of the proposed algorithm, only the magnitude factor among four real factors is used because the factor exhibits distinct characteristics based on fault conditions as mentioned in Sect. 2. In conclusion, the values of $A_{l}$ from the corresponding DC/DC converter $\# l^{*}$ are transferred to the control center to identify fault section.

\subsubsection{Decision stage: identification of fault type}

In the decision stage, the transferred data groups for $S_{l}$ and $A_{l}$ are analyzed in the control center to identify the fault section in the LVDC distribution system. To this end, the identifying step for fault type is conducted as the preceding step through four sub-steps. The first sub-step involves determining $\# l^{*}$ max with the maximum value of $A_{l}$ $\left(A_{\max }\right)$. Second, it is confirmed as to whether all values of $S_{l}$ correspond to ' 1 ' to check the fault type. If a $S_{l}$ with ' 0 ' exists, then it is identified a PTN fault and moves to the final sub-step. Otherwise, the third sub-step is implemented. In the third sub-step, the pattern of a series of $A_{l}$ is analyzed with $\# l^{*}$ max as the center. If (9) is satisfied, a fault type is identified as a PTP fault and the final sub-step is ignored. If not, it is identified as a PTG fault. The $d_{l}$ in (9) corresponds to the distance from $\# l^{*}{ }_{\text {max }}$ to $\# l^{*}$. When a fault type is identified as a PTG fault or a PTN fault, the faulty pole is determined as the pole connected with $\# l^{*}{ }_{\text {max }}$.

$A_{l} \propto 1 / d_{l}$

\subsubsection{Decision stage: identification of fault section}

In this section, the precise fault section is identified as the section on the left side or right side of $\# l_{\text {max }}^{*}$. It proceeds in different methods based on the fault type.

In the case of a PTP fault, the exact fault section is easily identified by comparing the values of $A_{l}$ on the left side and the right side $\left(A_{\max -1}, A_{\max +1}\right)$. If (10) is satisfied, then the left section of $\# l^{*}$ max is identified as the fault section and vice versa as:

$A_{\max -1}>A_{\max +1}$

Conversely, in the case of a PTG or a PTN fault, the method considering the characteristics in Sect. 2.2 is applied. Although a traveling wave by a PTG or PTN fault spreads to both sides, it is mostly disturbed by branches connected to the faulted pole. Naturally, a larger number of $\# l^{*}$ exists in the side of the fault section with $\# l^{*}{ }_{\max }$ as the center when compared to the opposite side. Thus, the exact fault section is identified by comparing the numbers of $\# l^{*}$ on both sides under a PTG or PTN fault.

\section{Simulation results}

\subsection{Simulation conditions}

In order to verify the performance of the proposed technique for identifying the fault section in a ring-type LVDC distribution system, a test system is modeled by using the EMTP as shown in Fig. 7. The test system includes eight load groups wherein the capacity and connection method to the distribution line are determined to consider the voltage unbalance problem in a bipolar LVDC system. DC/DC converter supplies each load group, and its

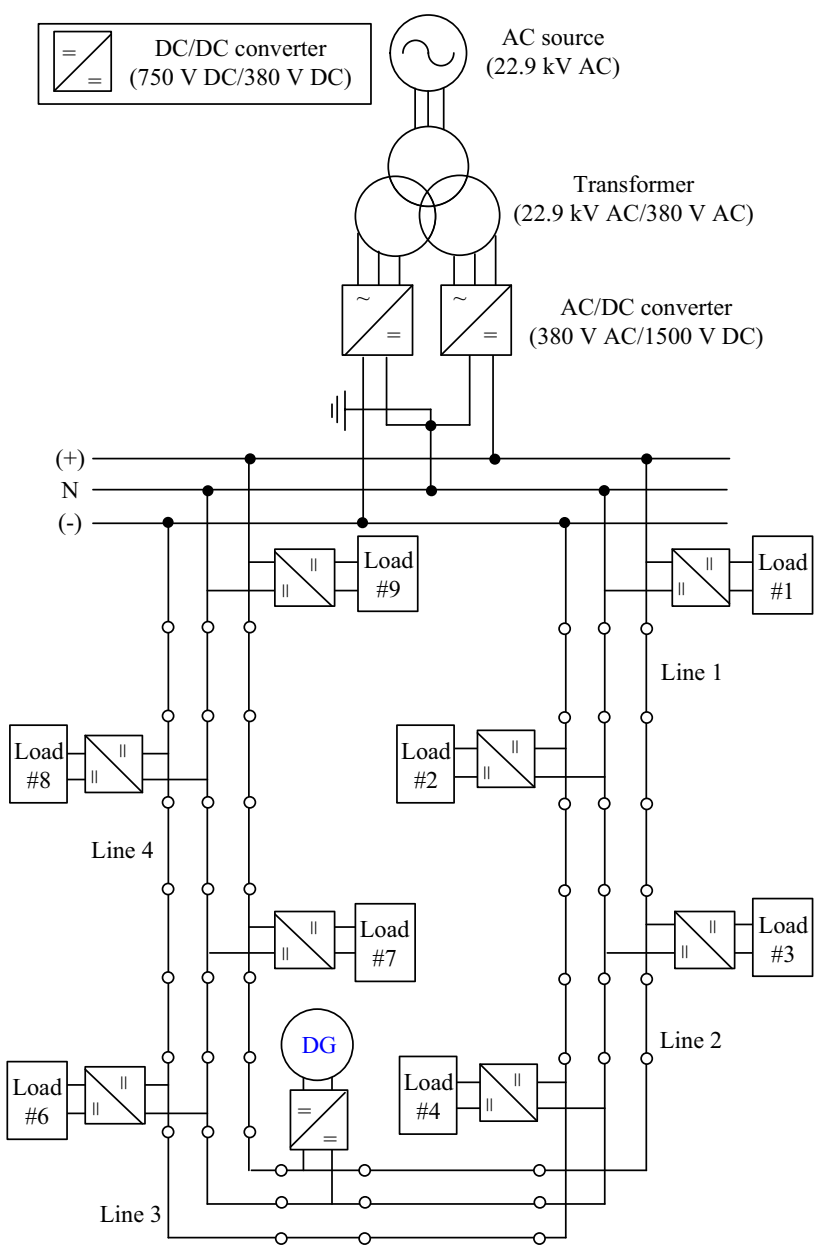

Fig. 7 Schematic diagram of test system 
switching frequency corresponds to $10 \mathrm{kHz}$. All the converters in the test system are assumed as possessing the protective function for semiconductor switches such that these switches are turned off immediately when an overcurrent is detected in the converters. Moreover, a DG is interconnected with the test system as shown in Fig. 7. DC/ DC converter functions as an interface between LVDC distribution system and DG as well. The capacitive discharge current is dominantly dependent on capacitor of DC/DC converter as discussed in Sect. 2 so that the existence of DG is uninfluential in the proposed technique. In conclusion, the proposed technique is applicable to LVDC distribution system interconnected with DGs and it is also verified by the simulations in this section. The detailed conditions of the test system are summarized in Table 3.

The simulations are conducted based on various fault conditions such as fault type and fault section. Furthermore, the fault resistance is considered as the fault condition in the case of a PTG fault. Each simulation lasts for $1 \mathrm{~s}$, and the fault occurs at $0.5 \mathrm{~s}$. The capacitive discharge currents to be measured were analyzed in EMTP, and a group of data to be transferred is analyzed in MATLAB ${ }^{\circledR}$ to identify the fault section in the test system. Furthermore, a comparison with the existing methods is performed to highlight the innovations of the proposed technique.

\subsection{Application of the conventional methods}

This section discusses the problems when the conventional methods discussed in Sect. 3.1 are applied to a ringtype LVDC distribution system. The most typical problem in the conventional methods involves requiring additional sensors when compared with the proposed technique. That is because at least three sensors must be installed for each measuring point while only a sensor is necessary for each DC/DC converter in the proposed technique. This problem

Table 3 Simulation conditions [34]

\begin{tabular}{lll}
\hline Parameter & & Input value \\
\hline Distribution line & Line constant & $R=0.164 \Omega / \mathrm{km}$ \\
& & $L=0.26 \Omega / \mathrm{km}$ \\
& Total length & $5000 \mathrm{~m}(500 \mathrm{~m}$ for each) \\
Load & Type & Purely resistive \\
& Capacity & $360 \mathrm{~kW}(40 \mathrm{~kW}$ for each) \\
AC/DC converter & Input reactance & $1.5 \mathrm{mH}$ \\
& DC-link & $5000 \mu \mathrm{F}$ \\
DC/DC converter & Input capacitance & \\
& LC filter $\quad L$ & $1000 \mu \mathrm{F}$ \\
& & $C$
\end{tabular}

is especially severe for the method to use current direction. Furthermore, other conventional methods involves additional problems.

First, the method based on voltage along the feeder can result in an estimation error because of the characteristics resulting from a fault. Figure 8 shows the simulation results for changes in voltage level in the section that is connected with DC/DC converter when a $(+)$ PTN fault occurs at@2 as shown in Fig. 7. This method identifies a fault section by measuring as to which section possesses the minimum voltage level. Nevertheless, as shown in Fig. 8, the voltage level typically decreases to zero with time. Thus, the estimation error will occur unless a general technique or standard is developed.

Next, in the method that uses the current difference, the capacitive discharge current from DC/DC converter causes an estimation error as shown in Fig. 9. The figure shows the current difference between two sections connected with \#2 and with \#3 when a (+) PTN fault occurs at @2 in Fig. 7. As shown in Fig. 9, high current is caused by the

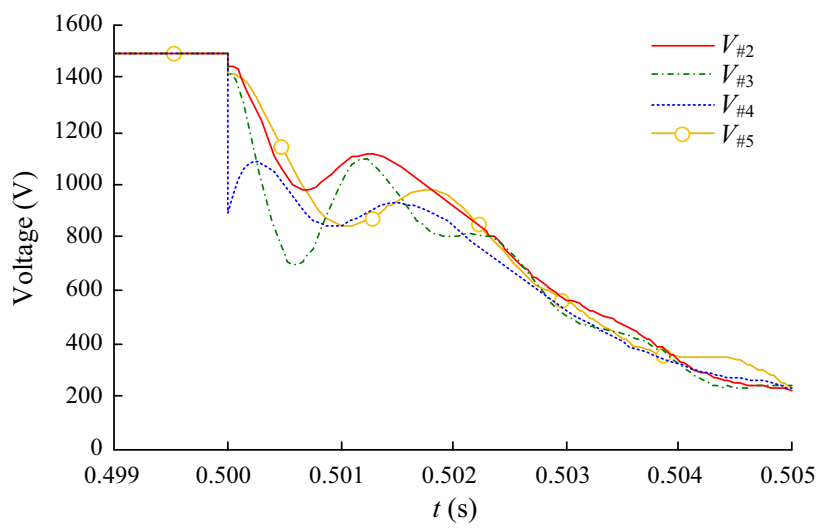

Fig. 8 Waveform of the voltage along feeder when (+) PTN fault occurs at@2

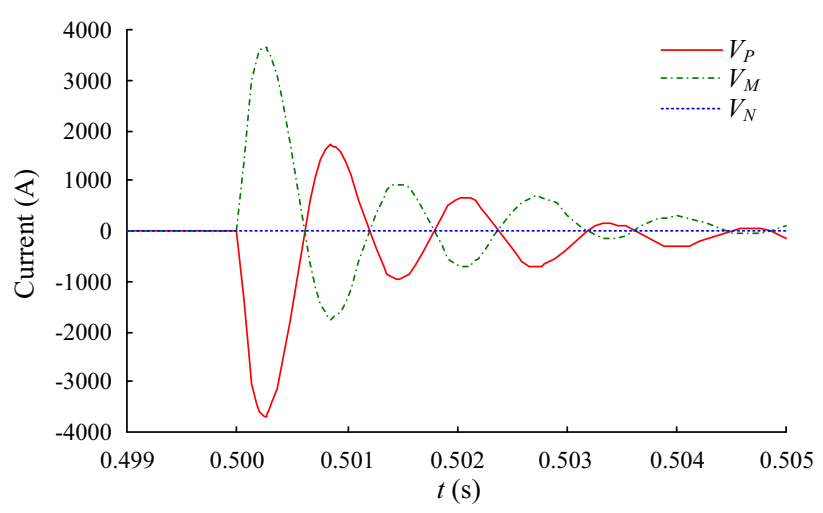

Fig. 9 Waveform of current difference when (+) PTN fault occurs at @2 
Table 4 Simulation results

\begin{tabular}{|c|c|c|c|c|c|c|c|c|c|c|c|c|c|c|c|c|c|c|c|}
\hline \multicolumn{2}{|l|}{ Fault type } & \multicolumn{9}{|c|}{ (+) PTG fault with $0.1 \Omega$} & \multicolumn{9}{|c|}{ (+) PTG fault with $10 \Omega$} \\
\hline Fault section & Output data & $\# 1$ & $\# 2$ & $\# 3$ & $\# 4$ & $\# 5$ & $\# 6$ & $\# 7$ & $\# 8$ & $\# 9$ & $\# 1$ & $\# 2$ & $\# 3$ & $\# 4$ & $\# 5$ & $\# 6$ & $\# 7$ & $\# 8$ & $\# 9$ \\
\hline \multirow{2}{*}{ Line @1 } & $S_{l}$ & 1 & 1 & 1 & 1 & & & & & & 1 & 1 & 1 & & & & & & \\
\hline & $A_{l}$ & 2000 & 820 & 1100 & 500 & & & & & & 60 & 20 & 23 & & & & & & \\
\hline \multirow{2}{*}{ Line @2 } & $S_{l}$ & & 1 & 1 & 1 & 1 & 1 & & & & & 1 & 1 & 1 & 1 & & & & \\
\hline & $A_{l}$ & & 670 & 1600 & 650 & 1100 & 520 & & & & & 15 & 65 & 20 & 28 & & & & \\
\hline \multirow{2}{*}{ Line@3 } & $S_{l}$ & & & & 1 & 1 & 1 & 1 & 1 & & & & & 1 & 1 & 1 & 1 & & \\
\hline & $A_{l}$ & & & & 230 & 1400 & 620 & 1100 & 450 & & & & & 15 & 65 & 20 & 25 & & \\
\hline \multirow{2}{*}{ Line@4 } & $S_{l}$ & & & & & & 1 & 1 & 1 & 1 & & & & & & 1 & 1 & 1 & 1 \\
\hline & $A_{l}$ & & & & & & 670 & 1800 & 770 & 800 & & & & & & 15 & 63 & 20 & 27 \\
\hline \multicolumn{2}{|l|}{ Fault type } & \multicolumn{9}{|c|}{ (-) PTG fault with $0.1 \Omega$} & \multicolumn{9}{|c|}{ (+) PTN fault } \\
\hline Fault section & Output data & $\# 1$ & $\# 2$ & $\# 3$ & $\# 4$ & $\# 5$ & $\# 6$ & $\# 7$ & $\# 8$ & $\# 9$ & $\# 1$ & $\# 2$ & $\# 3$ & $\# 4$ & $\# 5$ & $\# 6$ & $\# 7$ & $\# 8$ & $\# 9$ \\
\hline \multirow{2}{*}{ Line @1 } & $S_{l}$ & 1 & 1 & 1 & & & & & & 1 & 1 & o & 1 & 1 & & & & & \\
\hline & $A_{l}$ & 500 & 2200 & 630 & & & & & & 270 & 3500 & 910 & 1600 & 300 & & & & & \\
\hline \multirow{2}{*}{ Line @2 } & $S_{l}$ & 1 & 1 & 1 & 1 & 1 & & & & & & 1 & 1 & $\mathbf{0}$ & 1 & 1 & & & \\
\hline & $A_{l}$ & 450 & 1100 & 670 & 1900 & 550 & & & & & & 230 & 3800 & 720 & 1700 & 310 & & & \\
\hline \multirow{2}{*}{ Line @3 } & $S_{l}$ & & & 1 & 1 & 1 & 1 & 1 & & & & & & 1 & 1 & $\mathbf{0}$ & 1 & 1 & \\
\hline & $A_{l}$ & & & 460 & 1100 & 650 & 1900 & 540 & & & & & & 250 & 3800 & 720 & 1700 & 310 & \\
\hline \multirow{2}{*}{ Line@4 } & $S_{l}$ & 1 & & & & 1 & 1 & 1 & 1 & 1 & & & & & & 1 & 1 & 0 & 1 \\
\hline & $A_{l}$ & 210 & & & & 520 & 1200 & 720 & 2100 & 450 & & & & & & 250 & 3800 & 820 & 1500 \\
\hline \multicolumn{2}{|l|}{ Fault type } & \multicolumn{9}{|c|}{ (-) PTN fault } & \multicolumn{9}{|c|}{ PTP fault } \\
\hline Fault section & Output data & $\# 1$ & $\# 2$ & $\# 3$ & $\# 4$ & $\# 5$ & $\# 6$ & $\# 7$ & $\# 8$ & $\# 9$ & $\# 1$ & $\# 2$ & $\# 3$ & $\# 4$ & $\# 5$ & $\# 6$ & $\# 7$ & $\# 8$ & $\# 9$ \\
\hline \multirow{2}{*}{ Line @1 } & $S_{l}$ & 0 & 1 & 1 & & & & & & 1 & 1 & 1 & 1 & & & & & & \\
\hline & $A_{l}$ & 1100 & 3700 & 1000 & & & & & & 480 & 3800 & 4000 & 2300 & & & & & & \\
\hline \multirow{2}{*}{ Line @2 } & $S_{l}$ & 1 & 1 & $\mathbf{0}$ & 1 & 1 & & & & & 1 & 1 & 1 & 1 & 1 & & & & \\
\hline & $A_{l}$ & 240 & 1600 & 730 & 3800 & 270 & & & & & 1100 & 2300 & 4100 & 4000 & 2400 & & & & \\
\hline \multirow{2}{*}{ Line @3 } & $S_{l}$ & & & 1 & 1 & $\mathbf{0}$ & 1 & 1 & & & & & & 1 & 1 & 1 & 1 & 1 & \\
\hline & $A_{l}$ & & & 230 & 1600 & 730 & 3800 & 250 & & & & & & 2400 & 3900 & 4000 & 2600 & 1700 & \\
\hline \multirow{2}{*}{ Line @4 } & $S_{l}$ & & & & & 1 & 1 & 0 & 1 & 1 & & & & & & 1 & 1 & 1 & 1 \\
\hline & $A_{l}$ & & & & & 310 & 1700 & 740 & 3700 & 190 & & & & & & 2400 & 4000 & 4200 & 1900 \\
\hline
\end{tabular}

Note: The bold value means the highest A in each simulation case. This value can be the standard to distinguish fault types and identify fault section in the proposed algorithm

capacitive discharge current although the section that should be measured is healthy.

\subsection{Simulation results based on fault conditions}

This section shows the simulation results based on fault conditions to verify the performance of the proposed technique. All the simulation results are arranged as shown in Table 4. Figures 10, 11 and 12 depict examples of simulation results, presenting the waveform of capacitive discharge current from a DC/DC converter and the corresponding output data. The three figures correspond to real simulation results by using EMTP and show the manner in which the proposed algorithm proceeds. In Figs. 10, 11 and $12, i_{c d}$ presents the waveform of capacitive discharge current from \#3 in which the reducing part and identifying part 


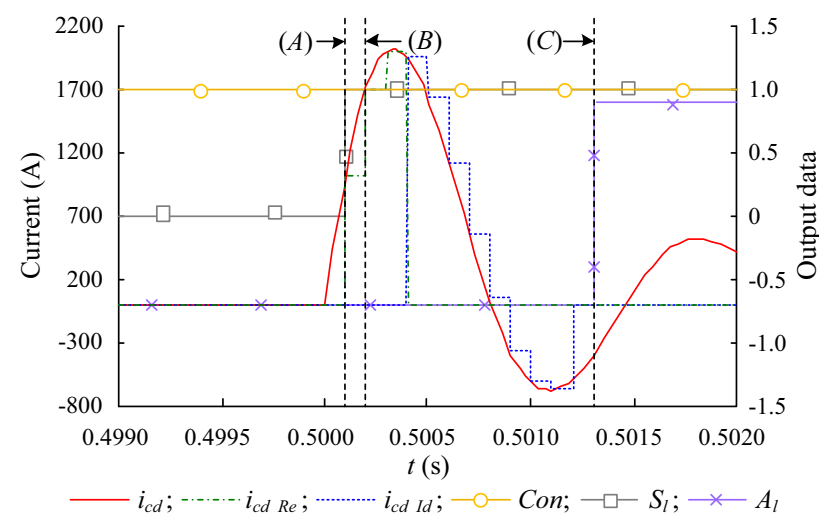

Fig. 10 Waveform of capacitive discharge current from \#3 when a (+) PTG fault occurs at @ 2 and the corresponding output data

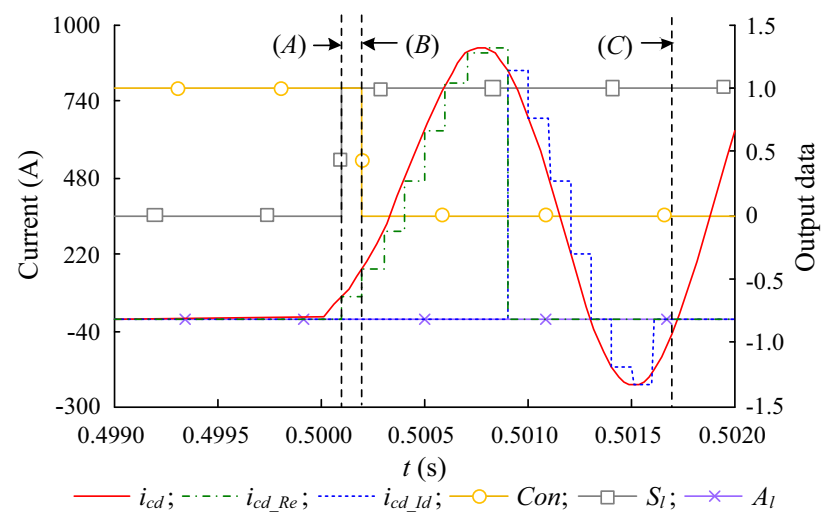

Fig. 11 Waveform of capacitive discharge current from \#1 when a (+) PTG fault occurs at @2 and the corresponding output data

are represented as $i_{c d_{-} R e}$ and $\mathrm{i}_{c d_{-} I}$, respectively. Next, Con denotes a factor to estimate the convexity of capacitive discharge current. If (4) is satisfied, presenting convex up, Con corresponds to ' 1 '. If not, Con corresponds to zero. Finally, $S_{l}$ and $A_{l}$ correspond to the output data from the corresponding current. Each waveform exhibits significant changes at the following three important time: (a) corresponds to the time to decide $S_{l}$ by using di/d $t$, (b) corresponds to when Con is determined by $\mathrm{d}^{2} i / \mathrm{d} t^{2}$, and (c) corresponds to the instant to output $A_{l}$ from identifying part.

The first two figures, Figs. 10 and 11, present the waveform of capacitive discharge current when a (+) PTG fault occurs at @2 in the test system. As shown in Fig. 10, Con corresponds to the value of ' 1 ' such that the output data $S_{l}$ and $A_{l}$ from \#3 are used to identify the fault section as shown in Table 4. In the case of the output data from \#1 as shown in Fig. 11, they are ignored because Con corresponds to zero. The last figure (Fig. 12) presents the waveform of capacitive discharge current from \#6 when (+) PTN fault occurs at @ 3 in the test system. As shown in Fig. 12, it is confirmed that the direction of capacitive

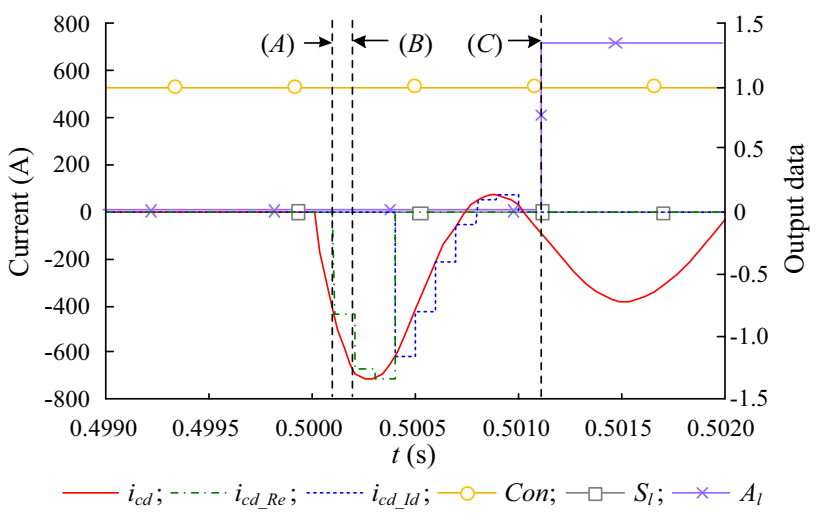

Fig. 12 Waveform of capacitive discharge current from \#6 when a (+) PTN fault occurs at @ 3 and the corresponding output data

discharge current is reversed under a PTN fault through the zero value of $S_{l}$ as described in Sects. 2.2 and 3.3.3.

In Table 4, it is verified that the fault section is accurately identified irrespective of fault conditions through the decision stage of the proposed technique. First, with respect to the aspect of fault type identification, it is possible to distinguish the fault type based on the pattern of $A_{l}$ and the value of $S_{l}$. In the case of a PTG fault, $A_{l}$ is not inversely proportional to $d_{f}$ and all the $S_{l}$ exhibit values corresponding to ' 1 '. However, in the case of a PTP fault, $A_{l}$ is inversely proportional to $d_{f}$ and all the $S_{l}$ simultaneously exhibit values corresponding to ' 1 '. Additionally, there is $S_{l}$ with the zero value in the case of the PTN fault. Furthermore, the proposed algorithm can identify the fault section based on fault type. In the case of a PTG fault and a PTN fault, it is confirmed that the number of $\# l^{*}$ on the side of fault section is larger than on the opposite side in Table 4. Moreover, the fault section is also identified under a PTP fault by comparing $A_{\max -1}$ with $A_{\max +1}$. Additionally, it is confirmed that the proposed technique identifies the precise fault section even in the case of a PTG fault with a slightly high impedance as shown in Table 4. In conclusion, it is verified that the proposed algorithm identifies the fault section in a ring-type LVDC distribution system.

\section{Conclusion}

This study proposed a technique to identify the fault section of a ring-type LVDC distribution system by using capacitive discharge currents from DC/DC converters. The current is more suitable for gathering information with respect to a fault than that from the AC/DC converter because DC/DC converters are distributed throughout the entire system. Additionally, it is not necessary to rapidly cut off the fault current from a DC/DC converter. 
Furthermore, the capacitive discharge current possesses distinct characteristics based on the fault conditions including the fault type and location.

In order to analyze the capacitive discharge current accurately and quickly, several considerations are applied with respect to signal processing using Prony's method in the proposed technique. For this purpose, only the data of DC/DC converters adjacent with fault location is used in terms of convexity criteria. Furthermore, the identifying part of the capacitive discharge current is used to decrease the number of sampled values in Prony's method, and this reduces the signal processing time. Given these aspects, the proposed technique includes two stages, namely an analysis stage at each DC/DC converter and a decision stage at the main computer. Essential data $\left(A_{l}\right.$ and $\left.S_{l}\right)$ are output in the analysis stage. This is followed by identifying the fault type and fault section by using $A_{l}$ and $S_{l}$ in the decision stage. The proposed technique is verified through various case study simulations by using the EMTP and MATLAB ${ }^{\circledR}$ that considers all the normal fault conditions in a test system and even includes DG. In conclusion, the proposed technique can be used to rapidly identify the fault section in an LVDC distribution system, and this improves its reliability.

Acknowledgements This work was supported by Human Resources Program in Energy Technology of the Korea Institute of Energy Technology Evaluation and Planning (KETEP), granted financial resource from the Ministry of Trade, Industry \& Energy, Republic of Korea. (No. 20164030200980) and by the National Research Foundation of Korea (NRF) grant funded by the Korea government (MSIP) (No. 2015R1A2A1A10052459).

Open Access This article is distributed under the terms of the Creative Commons Attribution 4.0 International License (http:// creativecommons.org/licenses/by/4.0/), which permits unrestricted use, distribution, and reproduction in any medium, provided you give appropriate credit to the original author(s) and the source, provide a link to the Creative Commons license, and indicate if changes were made.

\section{References}

[1] Oh YS, Han J, Gwon GH et al (2015) Development of fault detector for series arc fault in low voltage DC distribution system using wavelet singular value decomposition and state diagram. J Electr Eng Technol 10(3):766-776

[2] Byeon G, Hwang CS, Jeon JH et al (2015) Complementary power control of the bipolar-type low voltage DC distribution system. J Electr Eng Technol 10(3):786-794

[3] Kyei J (2001) Analysis and design of power acceptability curves for industrial loads. M.S. thesis, Arizona State University

[4] IEEE Standard 446 (1995) IEEE recommended practice for emergency and standby power systems for industrial and commercial applications
[5] Terzija V, Radojevic ZM, Preston G (2015) Flexible synchronized measurement technology-based fault locator. IEEE Trans Smart Grid 6(2):866-873

[6] Terzija V, Preston G, Stanojevic V et al (2015) Synchronized measurements-based algorithm for short transmission line fault analysis. IEEE Trans Smart Grid 6(6):2639-2648

[7] IEC 61660-1 (1997) Short-circuit currents in DC auxiliary installations in power plants and substations-part 1: calculation of short-circuit curernts

[8] Yang J, Fletcher JE, O'Reilly J (2010) Multiterminal DC wind farm collection grid internal fault analysis and protection design. IEEE Trans Power Deliv 25(4):2308-2318

[9] Xu MM, Xiao LY, Wang HF (2013) A Prony-based method of locating short-circuit fault in DC distribution system. In: Proceedings of 2nd IET renewable power generation conference, Beijing, China, 9-11 Sep., 2013, pp 1-4

[10] Rezmer J, Sikorski T (2011) Signal processing method for faults location in DC transmission system. In: Proceedings of 10th international conference on environment and electrical engineering, Rome, Italy, 8-11 May, 2011, pp 1-4

[11] Yang J, Fletcher JE, O'Reilly J (2012) Short-circuit and ground fault analyses and location in VSC-based DC network cables. IEEE Trans Ind Electron 59(10):3827-3837

[12] Emhemed AAS, Burt GM (2014) An advanced protection scheme for enabling an LVDC last mile distribution network. IEEE Trans Smart Grid 5(5):2602-2609

[13] Hebner R (2011) DC Protection, Electric ship research and development consortium for the office of naval research, Technical Report, Dec 192011

[14] Vimalraj S, Somasundaram P (2014) Fault detection, isolation and identification of fault location in low-voltage DC ring bus microgrid system. Int $\mathrm{J}$ Adv Res Electr Electron Instrum Eng 3(2):570-582

[15] Park JD (2015) Ground fault detection and location for ungrounded DC traction power systems. IEEE Trans Veh Technol 64(12):5667-5676

[16] Mohanty R, Balaji USM, Pradhan AK (2016) An accurate noniterative fault-location technique for low-voltage DC microgrid. IEEE Trans. Power Deliv 31(2):475-481

[17] Park JD, Candelaria J, Ma L et al (2013) DC ring-bus microgrid fault protection and identification of fault location. IEEE Trans Power Deliv 28(4):2574-2584

[18] Mora-Florez J, Melendez J, Carrillo-Caicedo G (2008) Comparison of impedance based fault location methods for power distribution systems. Electr Power Syst Res 78(4):657-666

[19] Apostolov A, Mysore P, Sidhu T et al (2012) An update to protection issues during system restoration. In: IEEE power and energy society general meeting, San Diego, USA, 22-26 July, 2012, 4 pp

[20] Mirzaei M, Moazami MZAAKE et al (2009) Review of fault location methods for distribution power system. Aust J Basic Appl Sci 3(3):2670-2676

[21] Dwivedi A, Yu X (2011) Fault location in radial distribution lines using traveling waves and network theory. In: Proceedings of IEEE international symposium on industrial electronics, Gdansk, Poland, 27-30 June, 2011, 6 pp

[22] Jahromi AT, Wolfs P, Islam S (2015) Traveling wave fault location in rural radial distribution networks to reduce wild fire risk. In: Proceedings of Australasian universities power engineering conference, Wollongong, Australia, 27-30 Sept, 2015, 6 $\mathrm{pp}$

[23] Sadeh J, Bakhshizadeh E, Kazemzadeh R (2013) A new fault location algorithm for radial distribution systems using modal analysis. Electr Power Energy Syst 45(1):271-278 
[24] Noh CH, Gwon GH, Song JI et al (2016) An analysis on fault response characteristics in low voltage DC distribution system. Trans Korean Inst Electr Eng 65(6):911-917

[25] Meghwani A, Srivastava SC, Chakrabarti (2016) A non-unit protection scheme for DC microgrid based on local measurements. IEEE Trans Power Deliv 32(1):172-181

[26] Patil G, Satarkar MFAR (2014) Autonomous protection of low voltage DC microgrid. In: Proceedings of international conference on power, automation and communication, Amravati, India, 6-8 Oct., 2014, 5 pp

[27] Tseng KJ, Luo C (2014) Power electronic-based protection for direct-current power distribution in micro-grids. In: Proceedings of the international power electronics conference, Hiroshima, Japan, 18-21 May, 2014, 7 pp

[28] Qi L, Liang J (2015) Design issues and practical application challenges of DC shipboard distribution system protection. In: Proceedings of IEEE electric ship technologies symposium, Alexandria, Egypt, 21-24 June, 2015, 6 pp

[29] Toshiba Corporation (2004) Instruction manual directional overcurrent protection relay GRD 140(6F2S0758-0.7)

[30] LSIS Corporation (2014) Digital Integrated Protection \& Monitoring Equipment(X-GIPAM technical report)

[31] Jalali D, Moslemi N (2005) Fault location for radial distribution systems using fault generated high-frequency transients and wavelet analysis. In: Proceedings of CIRED 2005-18th international conference on electricity distribution, Turin, USA, 6-9 June, 2005, 4 pp

[32] Qi L, Qian L, Woodruff S (2007) Prony analysis for power system transient harmonics. EURASIP J Adv Signal Process $1: 170$

[33] Chaari O, Bastard P, Meunier M (1995) Prony's method: an efficient tool for the analysis of earth fault currents in petersencoil-protected networks. IEEE Trans Power Deliv 10(3):1234-1241

[34] Peltoniemi P (2010) Phase voltage control and filtering in a converter-fed single-phase customer-end system of the LVDC distribution network. Dissertation, Lappeenranta University
Chul-Ho NOH received a B.S. degree from the College of Information and Communication Engineering, Sungkyunkwan University, Republic of Korea, in 2013. At present, he is enrolled in the combined master's and doctorate program. His research interests include power system transients, power quality, and protection schemes and coordination, especially in DC distribution systems.

Chul-Hwan KIM received B.S. and M.S. degrees in electrical engineering from Sungkyunkwan University, South Korea, in 1982 and 1984, respectively. He received a Ph.D. degree in electrical engineering from Sungkyunkwan University in 1990. In that same year, he joined Cheju National University, Cheju, South Korea, as a full-time lecturer. He was a visiting academic at the University of Bath, UK, in 1996, 1998, and 1999. Since March 1992, he has been a professor in the College of Information and Communication, Sungkyunkwan University, South Korea. His research interests include power system protection, the application of artificial intelligence to protection and control, the modeling/protection of underground cables, and EMTP software.

Gi-Hyeon GWON received his B.S. and M.S. degrees from the College of Information and Communication Engineering, Sungkyunkwan University, Korea, in 2012 and 2014, respectively. At present, he is working on his $\mathrm{Ph} . \mathrm{D}$. thesis. His research interests include power system transients, protection, and stability.

Yun-Sik OH received his B.S. and M.S. degrees from the College of Information and Communication Engineering, Sungkyunkwan University, Korea, in 2011 and 2013, respectively. At present, he is working on his $\mathrm{Ph} . \mathrm{D}$. thesis. His research interests include power system transients, protection, and stability, as well as renewable energy. 